\title{
A smartphone-based online tool for prehospital self-triage of COVID-19
}

\author{
Juan $\mathrm{Yu}^{1} \cdot$ Han-wen Zhang ${ }^{1} \cdot$ Yuan-kai Shao ${ }^{2} \cdot$ Yi Lei $^{1}{ }^{10} \cdot$ Huan Chen ${ }^{1} \cdot$ Zu-hui Pu ${ }^{1} \cdot$ Fan Lin $^{1} \cdot$ Hua-jian Xu ${ }^{1}$. \\ Yu-li Wang ${ }^{1} \cdot$ Chao Liang ${ }^{1} \cdot$ Li-hong Liu $^{1} \cdot$ Xia-jing Liu ${ }^{1} \cdot$ Wei-cai Dai ${ }^{1}$
}

Received: 26 February 2020 / Revised: 25 September 2020 / Accepted: 15 October 2020 / Published online: 16 November 2020

(c) Springer Nature Singapore Pte Ltd. 2020

\begin{abstract}
The COVID-19 epidemic has swept across China and spread to other countries. The rapid spreading of COVID-19 and panic combined with the lack of a hierarchical medical system in China have resulted in a huge number of hospital visiting which are overwhelming local medical system and increasing the incidence of cross infection. To meliorate this situation, we adopted the management concept of the system of Tiered Diagnosis and Treatment and developed an online tool for self-triage based on the mostly used multi-purpose smartphone app Wechat in China. This online tool helps people perform self-triage so that they can decide whether to quarantine at home or visit hospital. This tool further provides instructions for home quarantine and help patients make an appointment online if hospital visiting suggested. This smartphone application can reduce the burden on hospitals without losing the truly COVID-19 patients and protect people from the danger of cross infection.
\end{abstract}

Keywords COVID-19 $\cdot$ Smartphone-based online tool $\cdot$ Prehospital $\cdot$ Self-triage

\section{Background}

The disease COVID-19 caused by the novel coronavirus SARS-CoV-2 (previously 2019-nCoV) has been spreading rapidly since the initial discovery in December of 2019. As of 24:00 on February 15, more than 70,000 confirmed cases have been reported in China [1]. In comparison to SARS and MERS, COVID-19 is characterized by several unique characteristics, such as strong virulence, longer

Juan Yu, Han-wen Zhang, and Yuan-kai Shao contributed equally to this work.

Yi Lei and Huan Chen contributed equally to this work.

Yi Lei

13602658583@163.com

1 Department of Radiology, Health Science Center, The First Affiliated Hospital of Shenzhen University, Shenzhen Second People's Hospital, 3002 SunGangXi Road, Shenzhen 518035, China

2 Department of Emergency, Health Science Center, The First Affiliated Hospital of Shenzhen University, Shenzhen Second People's Hospital, 3002 SunGangXi Road, Shenzhen 518035, China incubation period, and insensitivity to nucleic acid detection. Although many mild patients do not show severe symptoms at early stage of infection, they will soon start the inflammatory storm leading to multiple organ failure [2]. These characteristics of COVID-19 and our lack of counteractive measurements, coupled with the huge number of passenger journeys during the Chinese New Year period, have led to the rapid spreading of the disease which is exhausting local medical resources. All of these factors have brought great challenges to the prevention and control of the COVID-19 epidemic. According to Professor Zhong, a top Chinese expert on respiratory medicine who has contributed significantly to the combat against SARS, two necessary measures must be taken to ameliorate this situation: first, we need effective patient diversion to reduce the burden on the designated hospitals. Yet, the lack of a hierarchical medicine system in China has led a huge number of patients to visit a small number of major hospitals which is overwhelming these hospitals. Second, it is necessary to optimize admission process and hospital management to effectively prevent hospital-acquired infections and ensure the safety of medical staff and patients. We believe that we can adopted the concepts of the system of Tiered Diagnosis and Treatment which has achieved promising results in Singapore without 
affecting the local economy and people's daily life $[3,4]$. We developed a smartphone-based online tool to: (1) identify common flu patients, who can stay at home to conduct self-treatment instead of going to hospitals to increase the burden on hospitals and the chance of cross infection; (2) provide medical guidance for self-quarantine to those who have contact history with COVID-19 patients but show no symptoms; (3) identify symptomatic patients who have been in contact with someone already infected and instruct them to seek medical treatment in a timely manner and meanwhile simplify the admission procedures; (4) provide guidance to patients with special needs such as pregnant women and patients with other diseases; (5) provide doctor-provided online education of COVID-19, instructions of drug purchase and psychological counseling.

\section{Materials and methods}

1. The design of the WeChat mini program

A questionnaire was generated according to the latest seventh edition of $A$ rapid advice guideline for the diagnosis and treatment of 2019 novel coronavirus (2019-nCoV) infected pneumonia (standard version) [5] and this questionnaire was used to develop a WeChat mini program to evaluate patients. The logic diagram of diagnosis is shown in Fig. 1.

\section{Suggested measurements}

A, health (maximum possible) Self-quarantine for 14 days, monitor body temperature daily, wear a mask when going out, and avoid going to crowded indoor places if possible, and wash hands immediately when returning home. Once a history of epidemiological exposure is found, treat it as a suspected infected person.

$B$, common $f u$ Quarantine observation at home and monitor body temperature closely. When the body temperature exceeds $38.5^{\circ} \mathrm{C}$, take no more than four times a day of $0.5 \mathrm{~g}$ acetaminophen tablets or $0.3 \mathrm{~g}$ ibuprofen, with an interval of more than $4 \mathrm{~h}$. If fever accompanied by cough and occlusive runny nose, take CONTAC or Paracetamol (no co-administration with acetaminophen). If fever accompanied with cough, take potassium lignan sulfonate or dextromethorphan hydrobromide. When sore throat is obvious, take watermelon frost lozenge, Yinhuang buccal tablets, or ZhongSheng pills. For diarrhea, take montmorillonite powder and a low-fat, low-protein diet. Take good rest and drink plenty of water. If the fever is obvious (over $38{ }^{\circ} \mathrm{C}$ ) and sore throat and muscle soreness are obvious, oral administration of $75 \mathrm{mg}$ oseltamivir phosphate capsules (Tamiflu or Kewei), two times a day is recommended. Seek immediate consultation if persistent high fever (body temperature exceeds $39^{\circ} \mathrm{C}$ or difficulty in reducing fever) or worsening symptoms.

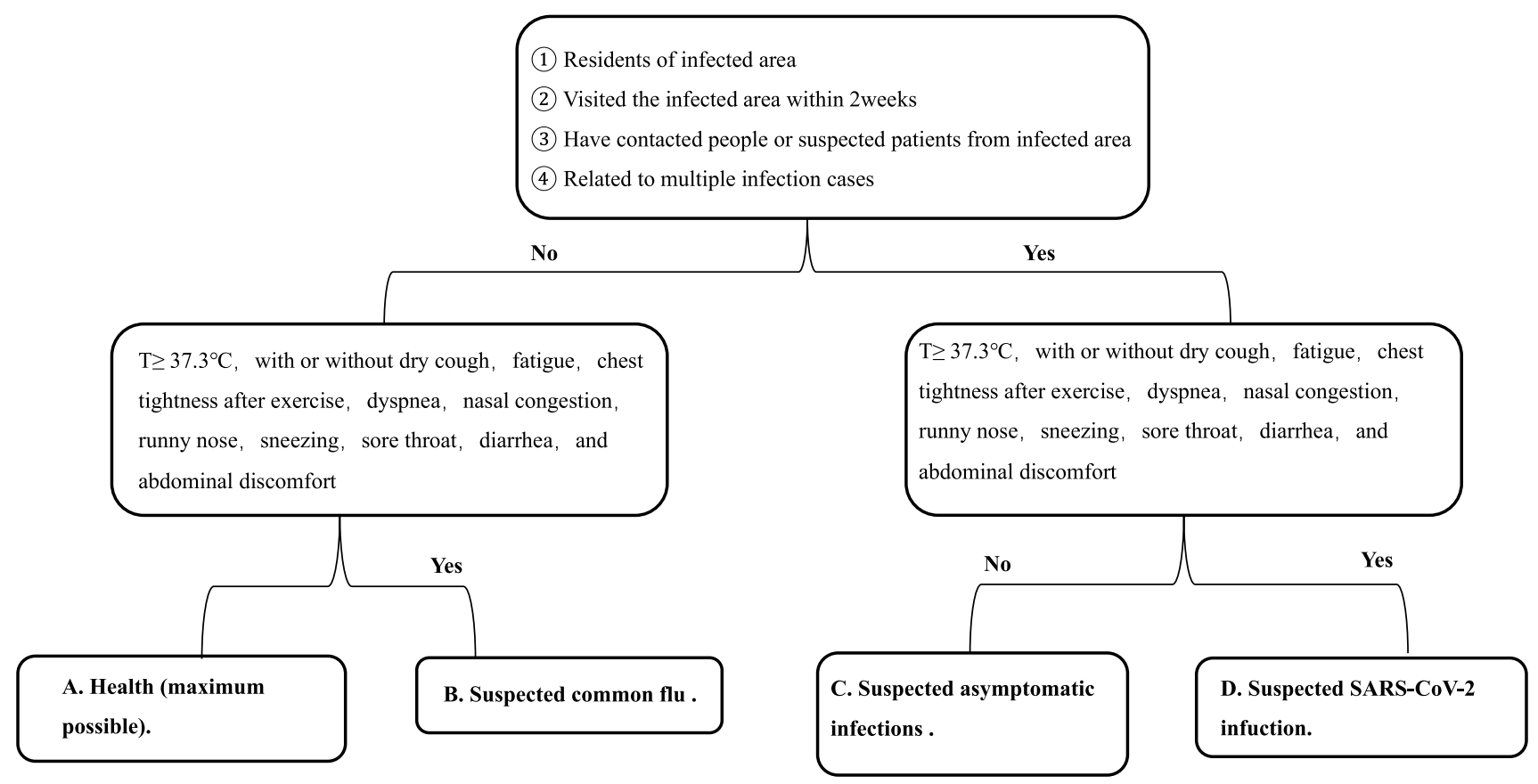

Fig. 1 Flowchart diagram of the diagnosis process of the online tool. Box a-d represents four different triage types. Details are in the method part 
Pregnant women Consider online consultation or go to an obstetrics and gynecology clinic and take medicines suitable for pregnant women. Take prevention measurements such as wearing mask when going out is necessary. Do not go out if unnecessary. Avoid touching eyes, mouth and nose, and minimize the time spent in crowds. Avoid contact with people or suspected patients from affected areas. Ensure enough sleep. Wash hands with clean running water and hand sanitizer when returning home. Conduct strict self-monitoring, and arrange inspection according to the doctor's instructions. Make an appointment in advance to minimize the time spent in the hospital, and pay attention to personal protection.

Children If under 5 years old or have high fever, advised to seek medical treatment in time. If choose to observe at home, pay close attention to body temperature, tiredness, breathing pattern and diet. When the body temperature exceeds $38.5^{\circ} \mathrm{C}$, take acetaminophen suspension (Tenolin) at a dose of $10 \mathrm{mg} / \mathrm{kg}$, or ibuprofen (MOTRIN) at a dose of $0.5 \mathrm{ml} / \mathrm{kg}$ with the maximum daily dose $40 \mathrm{mg} / \mathrm{kg}$. No more than four times of antipyretic drugs a day, with an interval of more than $4 \mathrm{~h}$. For diarrhea, take montmorillonite powder and a low-fat, low-protein diet. Take good rest and drink plenty of water. Seek immediate medical treatment if have persistent high fever or worsening symptoms, vomiting, wheezing, or coughing. High fever in children has the risk of febrile seizures, and body temperature need to be closely monitored.

If the patient has a history of complicated disease, such as diabetes, heart disease, chronic lung disease or other diseases, especially for people over 65 years old, see a doctor immediately.

$C$ Suspected asymptomatic infections. Designated isolation for more than 14 days to minimize the range of activities, perfect nucleic acid and chest CT examination.
Monitor their close contacts according to their whereabouts until the quarantine is lifted.

$D$ Suspected SARS-CoV-2 infection. Search the official website to check whether have been in a close contact using name and national ID number. It is recommended to go to the hospital to complete routine blood test, chest CT scan, antibody detection, and nucleic acid examination, and report to the community office and community health institutions for the record, monitor their close contacts.

2. Prototype interface was designed using the online design tool MODAO.

3. Functional implementation of the tool is through registering a public account on WeChat and developing the mini program using a special tool developed by WeChat.

\section{Results}

\section{A sample of the self-assessment page is shown below (Fig. 2a-f)}

Patients are assessed by clicking on a series of question-andanswer selections, which use easy-to-understand descriptions. The page design is simple and straightforward. Figure $2 \mathrm{a}$ includes basic information, including residence, name, age, gender, and itinerary. Figure $2 b$ is contact history. Figure $2 \mathrm{c}$ is the physical condition. Figure $2 \mathrm{~d}$ is a page for uploading available medical data. Figure $2 \mathrm{e}$ and $\mathrm{f}$ are two sample displays of the two results.

\section{Additional functions of the APP}

Online doctor consultation (Fig. 3): Patients can communicate with doctors online. For example, pregnant women

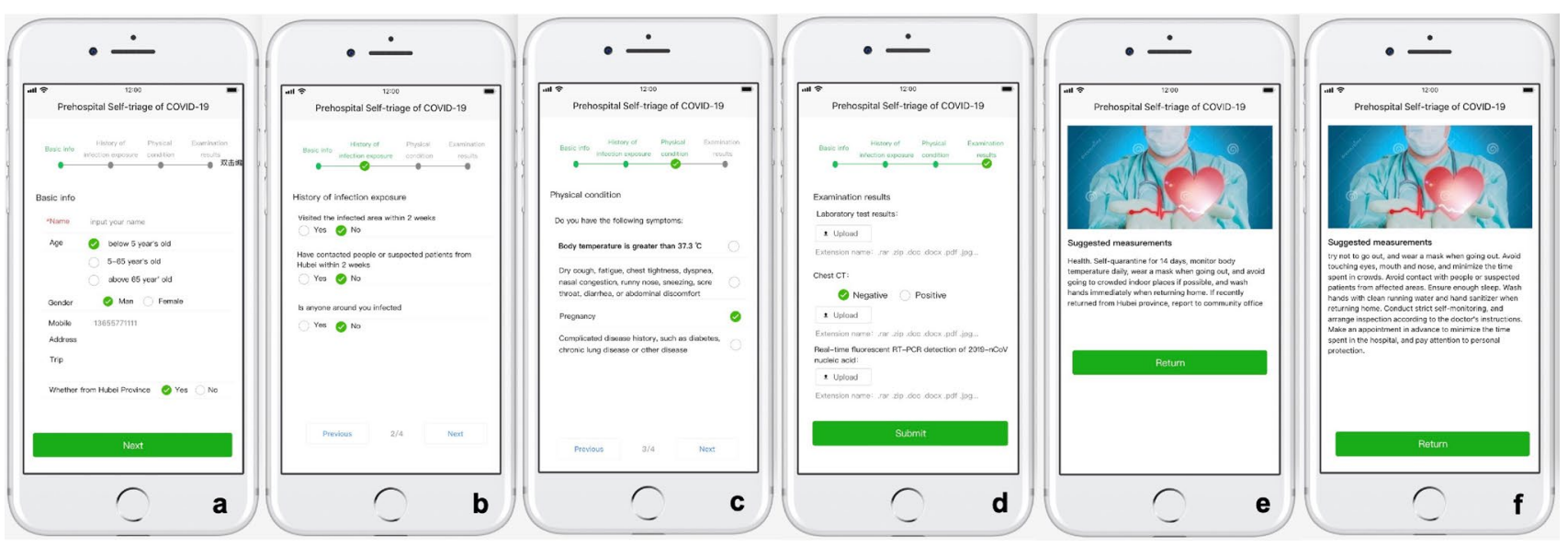

Fig. 2 Self-assessment page. a Basic information; b exposure history; $\mathbf{c}$ physical condition; d medical examination results; e display of example result $\mathrm{A} ; \mathbf{f}$ display of example result $\mathrm{B}$ 


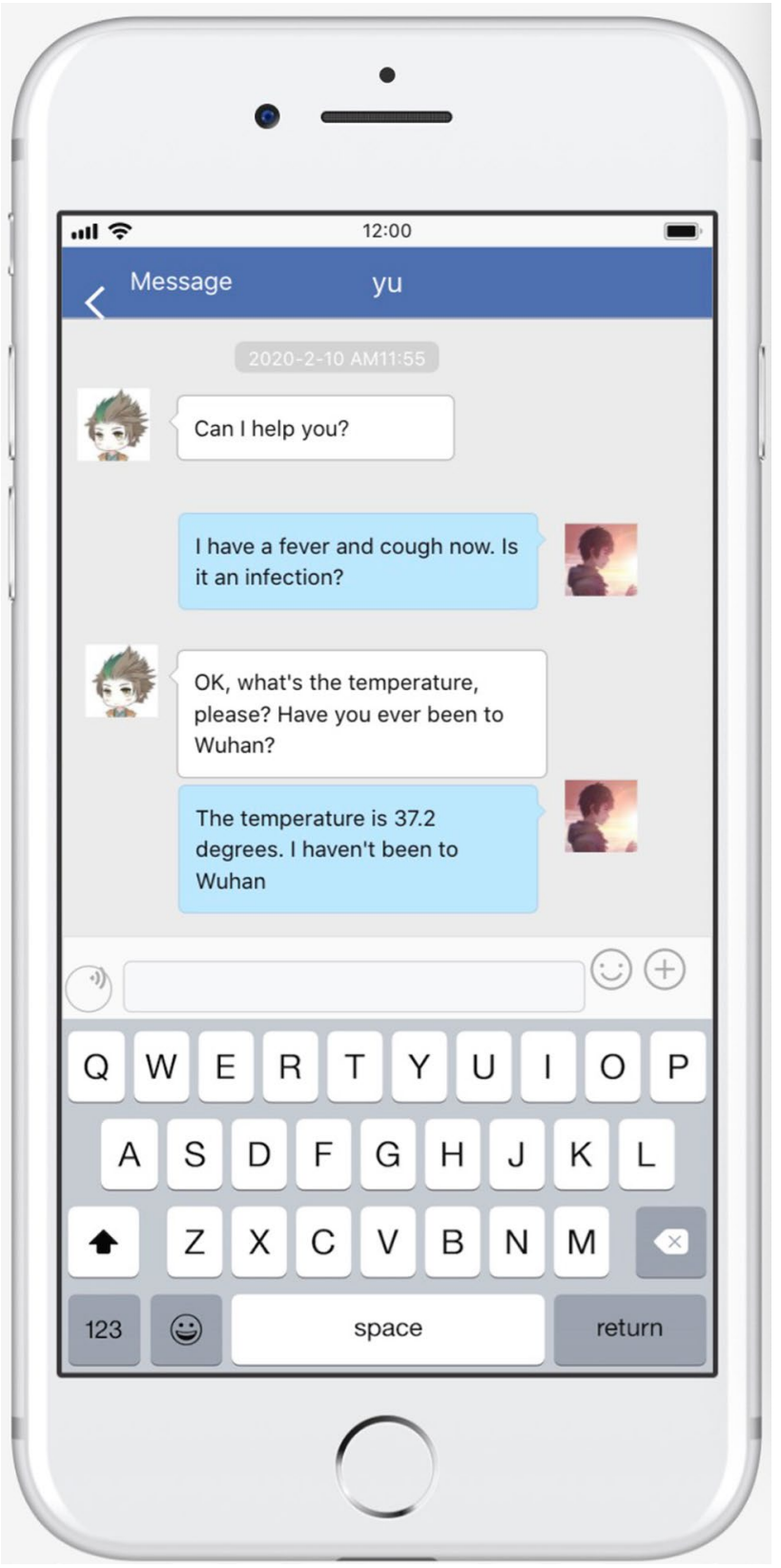

Fig. 3 Doctor consulting page, click the home task bar-select your favorite specialist-doctor consulting

can contact obstetricians and gynecologists with whom they have regular check-ups.

Other functions (Fig. 4).

1. Online drug purchase: patients can be redirected to other popular shopping platforms in China by clicking this page: JD.com, Alibaba's Tmall and other drug purchase platforms, which have doctors and pharmacists to review cases and provide guidance and help deliver drugs to home.

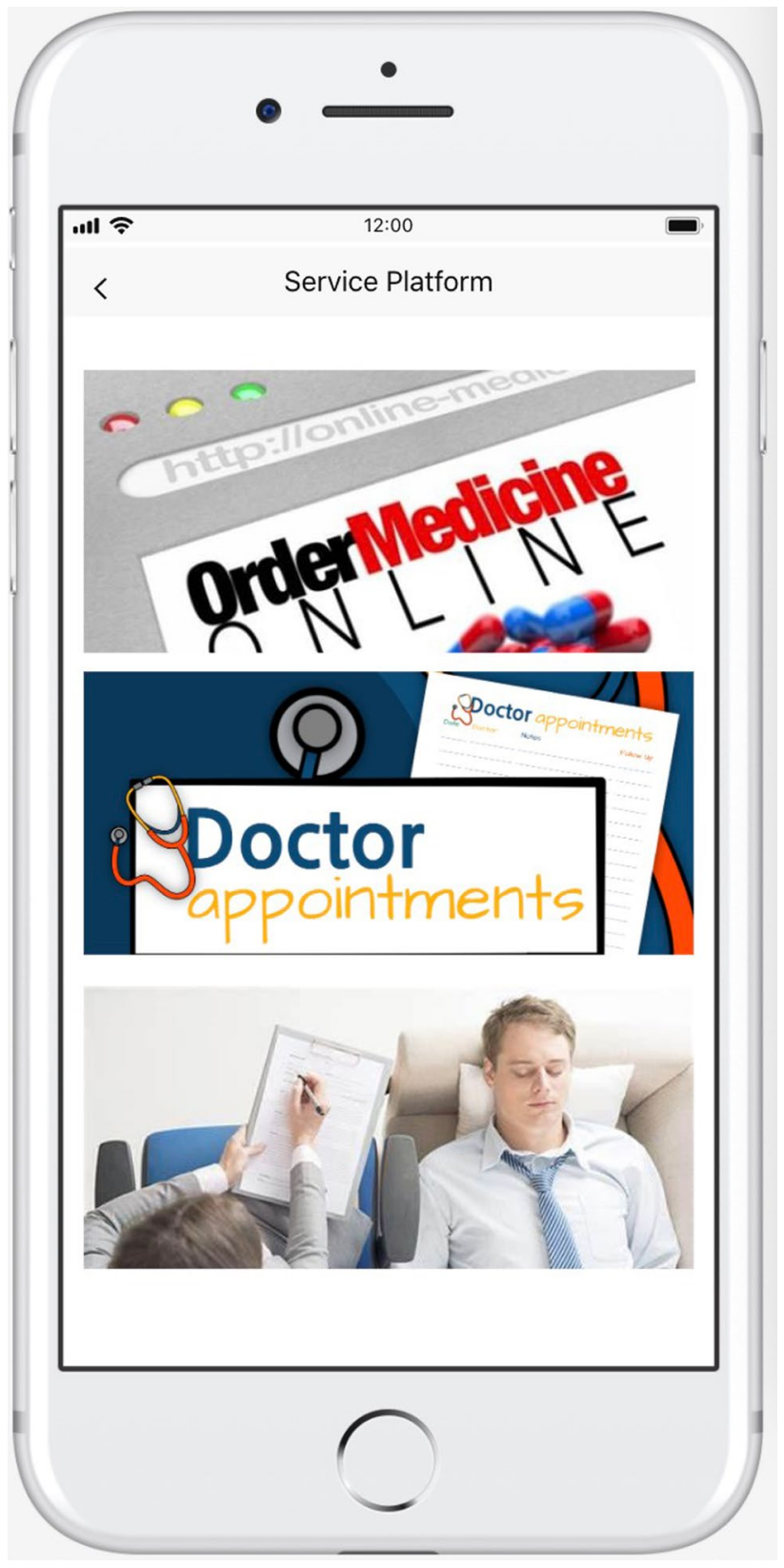

Fig. 4 The facilitating platform includes three sections: online drug purchase, appointment, and psychological consultation. After each click, users will be redirected to the relevant online platform

2. Online psychological consultation: redirect to the psychiatrist platform where many psychological experts provide free consultation specifically for the epidemic.

3. Online appointments with designated hospitals: the APP redirects to the website of the designated hospital nearest to the patient. Each hospital has an online booking platform on which patients can make appointment to avoid contact with paper documents at hospitals and minimize hospital staying. 
4. Online instructions and information from experts (Fig. 5): the amount of information from various sources is overwhelming and it is very difficult for patients to tell whether they are true. This online tool is maintained by doctors who can provide the most effective guidance for patients, including how to quarantine at home, how to travel, real-time epidemic reporting.

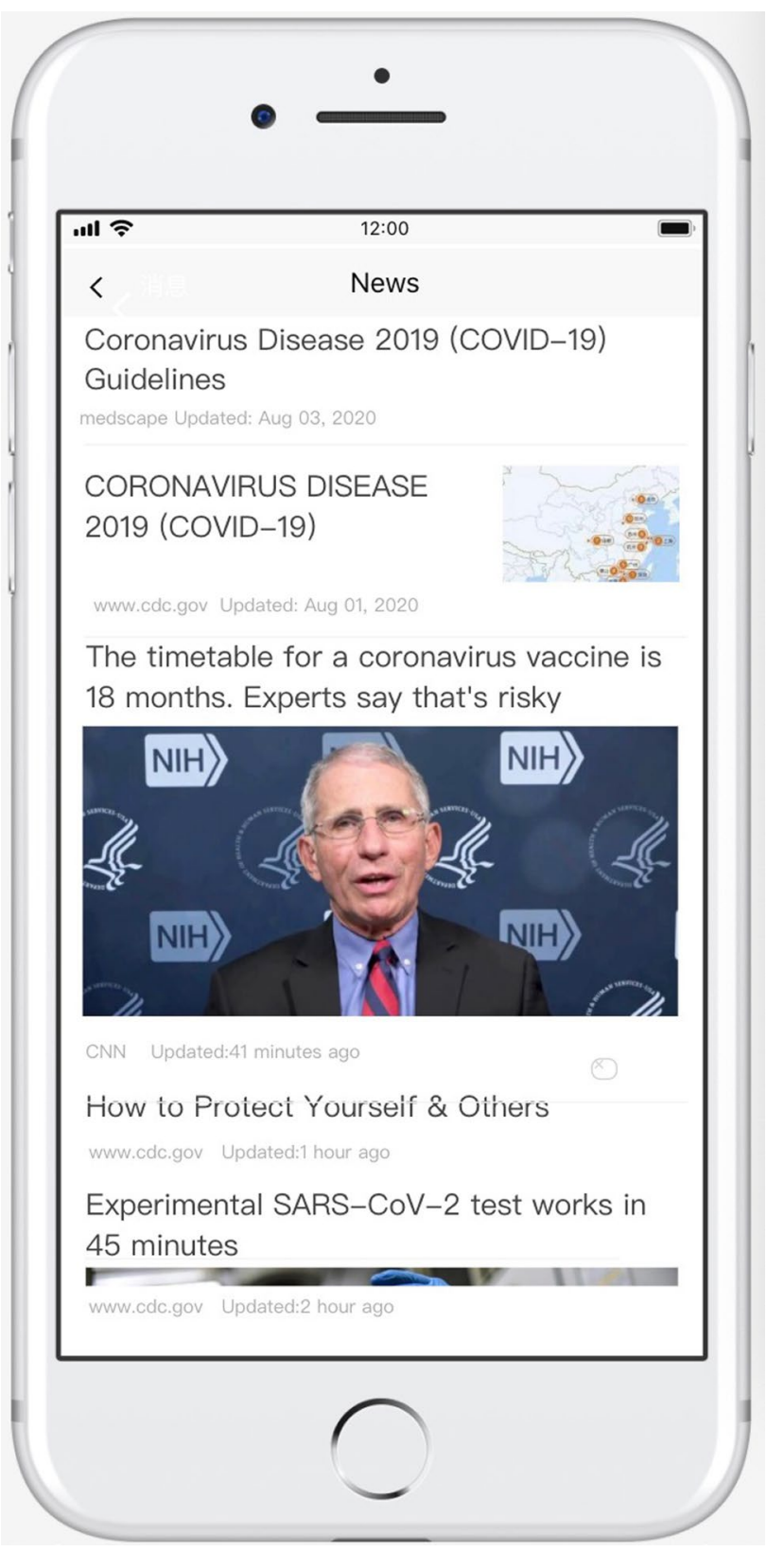

Fig. 5 Consulting service page. Real-time updated authoritative information and home travel guidance are filtered by doctors and pushed to users by the app

\section{Discussion}

The novel coronavirus SARS-CoV-2, which is causing the severe COVID-19 epidemic, is featured by strong virulence, long incubation period, and insensitivity to nucleic acid detection. Many mild patients quickly transition to severe disease status due to sudden inflammatory storm. The huge number of passenger journeys during the Chinese New Year period have accelerated the spreading of the disease. China's lack of hierarchical medical system led to all patients concentrated in major hospitals creating huge burden for these hospitals. According to Du et al. [6], there are 36 tertiary-A hospitals in Wuhan which are the top tier medical institutes based on the classification system of Chinese hospitals, with a total of 84,108 beds. Even with continuing aid from other hospitals all over the country, these hospitals are still not able to handle such huge number of COVID-19 patients. Shenzhen of Guangdong Province, where the authors are located, is also a large city. Currently, there are only 16 tertiary-A hospitals with 41,512 beds. However, recent census shows that the number of residents who have lived in Shenzhen for at least more than 1 month has been over 22 million. The imminent returning of people to work from the traditional spring festival holiday will further exacerbate the sever situation of lacking medical resources in Shenzhen. We believe that we can take advantage of the fast development of IT and internet services in recent years to mitigate this situation. Online services such as online office, consultation, shopping, appointment, and mobile payment are very popular in China. This study attempts to design a smartphone-based tool to provide people with a comprehensive and efficient online medical service to identify true COVID-19 patients and protect uninfected persons. WeChat is the most widely used multi-purpose messaging, social media and mobile payment app in China. The number of WeChat users already reached 1 billion. A tool which can be incorporated into WeChat can easily reach the majority of China's population. Moreover, compared to the recent software designed by Tsinghua University [7], our WeChat-based tool are convenient and simple for WeChat users to use with no difficulties. It also contains many functions in addition to self-triage, such as online consultation, information providing, etc. Thus, we believe that our WeChat-based app can contribute greatly to our combat against the COVID-19 epidemic. 


\section{Conclusions}

This smartphone-based online tool can help patients to perform self-triage at home, thus reducing panic and the incidence of hospital-acquired cross infection. It can also help us to easily detect and track down the occurrence of disease at the earliest time. Moreover, by functional implementation through the most popular app WeChat which has more than 1 billion users, this tool can quickly reach the major population of China. Our tool can help regions lacking medical resources contribute to our combat against the COVID-19 epedemic.

\section{References}

1. Ayittey FK, Ayittey MK, Chiwero NB, Kamasah JS, Dzuvor C. Economic impacts of Wuhan 2019-nCoV on China and the World. J Med Virol. 2020. https://doi.org/10.1002/jmv.25706 (published online ahead of print, $2020 \mathrm{Feb}$ 12).

2. Wang D, Hu B, Hu C, Zhu F, Liu X, Zhang J, et al. Clinical characteristics of 138 hospitalized patients with 2019 novel coronavirus-infected Pneumonia in Wuhan, China. JAMA. 2020. https ://doi.org/10.1001/jama.2020.1585 (published online ahead of print, 2020 Feb 7).

3. Anantharaman V. Impact of health care system interventions on emergency department utilization and overcrowding in Singapore. Int J Emerg Med. 2008;1(1):11-20.

4. Shi Y, Liu X, Kok SY, Jayanthi R, Liang S, Grace Y, et al. Threemonth real-time dengue forecast models: an early warning system for outbreak alerts and policy decision support in Singapore. Environ Health Perspect. 2016;124(9):1369-75.

5. Jin YH, Cai L, Cheng ZS, Cheng H, Deng T, Fan YP, et al. A rapid advice guideline for the diagnosis and treatment of 2019 novel coronavirus (2019-nCoV) infected pneumonia (standard version). Mil Med Res. 2020;7(1):4 (Published 2020 Feb 6).

6. Du Z, Wang L, Cauchemez S, Xu XK, Wang XW, Cowling BJ, et al. Risk for transportation of 2019 novel coronavirus disease from Wuhan to other cities in China. Emerg Infect Dis. 2020. https ://doi.org/10.3201/eid2605.200146 (published online ahead of print, 2020 May 17).

7. 2019-ncov.tsing care. https://2019-ncov.tsingcare.com/surve $\mathrm{y} \cdot \mathrm{html}$ ?from $=$.singlemessage $\&$ isappinstalled $=0 \&$ scene $=1 \&$ click time $=1581355546 \&$ enterid $=1581355546$

Publisher's Note Springer Nature remains neutral with regard to jurisdictional claims in published maps and institutional affiliations. 